\title{
Bioavailability of Two Tablet Formulations of a Single Dose of Moxifloxacin 400 mg: An Open-Label, Randomized, Two-Period Crossover Comparison in Healthy Mexican Adult Volunteers
}

Gabriel Mendoza-Tamayo ${ }^{1}$, Alejandra Rosete-Reyes ${ }^{1}$, Roberto Medina-Santillán', Jessica González-Bañuelos², Clara Espinosa-Martínez², Victoria Burke-Fraga ${ }^{2}$ and Mario González-de la Parra ${ }^{2 *}$

${ }^{1}$ CIF-BIOTEC, Médica Sur, S.A.B. de C.V., Mexico City, Mexico

${ }^{2}$ Biokinetics, S. A. de C. V., Mexico City, Mexico

\begin{abstract}
Moxifloxacin is a synthetic fluoroquinolone with a broad spectrum of antibacterial activity. It is indicated for the treatment of respiratory tract, skin and intra-abdominal infections.

The aim of this study was to compare the bioavailability and to determine the bioequivalence of a test and reference formulation of oral moxifloxacin $400 \mathrm{mg}$, administered as a tablet, and to generate data regarding the oral bioavailability of this drug in Mexican population.

This single-dose, randomized-sequence, open-label, two-period, crossover study was conducted on a total of 26 healthy Mexican adult subjects of both genders, with an eight-day washout period. Study formulations were administered after a 10-hour overnight fast. For pharmacokinetic analysis, blood samples were drawn at 0 (baseline), $0.25,0.5,0.75,1,1.5,2,3,4,5,6,8,12,24,48$ and 72 hours after administration. Plasma concentrations of moxifloxacin were determined using HPLC coupled with a fluorescence detector. The test and reference formulations were considered bioequivalent if the $90 \% \mathrm{Cls}$ for the geometric mean test/reference ratios were within a predetermined range of $80 \%$ to $125 \%$.
\end{abstract}

The $90 \%$ Cls for the geometric mean ratios of $\mathrm{C}_{\text {max }}, \mathrm{AUC}_{0-\mathrm{t}}$ and $\mathrm{AUC}_{0-\infty}$ were $88.67 \%$ to $108.70 \%, 97.44 \%$ to $102.50 \%$, and $97.70 \%$ to $104.82 \%$, respectively.

In this study a single dose of the test formulation met the regulatory requirements for assuming bioequivalence, based on the rate and extent of absorption.

Keywords: Moxifloxacin; Bioequivalence; Bioavailability; Pharmacokinetics; HPLC

\section{Introduction}

Moxifloxacin hydrochloride is a synthetic fluoroquinolone antibacterial agent with a broad spectrum of activity, encompassing Gram-negative and Gram-positive bacteria, indicated for the treatment of respiratory infections $[1,2]$.

Moxifloxacin is well absorbed following oral administration with an absolute bioavailability of $91.8 \%$. [3].

Its mean elimination half-life is approximately 12 hours under steady-state conditions [4].

Moxifloxacin shows linear pharmacokinetics after single doses ranging from 50 to $800 \mathrm{mg}$ [5] and its bioavailability is not affected by the concomitant intake of food [6].

The reference tablet formulation (Avelox ${ }^{\circledR}$, Bayer de México, S.A. de C.V, Mexico City, Mexico) is marketed in Mexico. Itwas selected as the reference formulation because it is included in the list of Drug Reference Medications issued by the Mexican Federal Commission for the Protection against Sanitary Risks (COFEPRIS). It is important to point out that the reference medications (formulations) are indicated in a list that is mandatory for bioequivalence studies performed in Mexico.

The test formulation (Zinolox $4 \mathrm{G}^{\circledR}$, Laboratorios Liomont, S. A. de C. V., Mexico City, Mexico) was selected because the sponsor of the present study wanted to obtain its marketing authorization in Mexico.
A search of PubMed, MEDLINE and Google data bases for literature published up to May of 2014, using the combination terms moxifloxacin, bioequivalence, bioavailability, pharmacokinetics, Mexico, Mexican and population, did not identify any published data concerning the bioavailability of oral moxifloxacin in the Mexican population.

Therefore, the aim of this study was to compare the bioavailability and to determine the bioequivalence of a test and reference formulation of oral moxifloxacin $400 \mathrm{mg}$ and to generate data regarding its bioavailability in the Mexican population for the purpose of obtaining the marketing authorization of the test formulation in Mexico.

\section{Subjects, Materials and Methods}

The study protocol (P455S026V006) and the informed-consent

*Corresponding author: Mario González-de la Parra, Privada Jesús del Monte No.77,Col. Cuajimalpa, 05000 Mexico City, Mexico, E-mail: mdelaparra@biokinetics.com.mx

Received September 25, 2014; Accepted October 30, 2014; Published November 02, 2014

Citation: Tamayo GM, Reyes AR, Santillán RM, Bañuelos JG, Martínez CE, et al (2014) Bioavailability of Two Tablet Formulations of a Single Dose of Moxifloxacin 400 mg: An Open-Label, Randomized, Two-Period Crossover Comparison in Healthy Mexican Adult Volunteers. J Bioequiv Availab 6: 197-201. doi:10.4172/ jbb.1000204

Copyright: @ 2014 Tamayo GM, et al. This is an open-access article distributed under the terms of the Creative Commons Attribution License, which permits unrestricted use, distribution, and reproduction in any medium, provided the original author and source are credited. 
Citation: Tamayo GM, Rosete A, Santillán RM, González J, Martínez CE, et al. (2014) Bioavailability of Two Tablet Formulations of a Single Dose of Moxifloxacin 400 mg: An Open-Label, Randomized, Two-Period Crossover Comparison in Healthy Mexican Adult Volunteers. J Bioequiv Availab 6: 197-201. doi:10.4172/jbb.1000204

form were approved by an independent ethics and research committee (Comité de Ética e Investigaciónpara Estudios en Humanos, Mexico City, Mexico) on October 10, 2012 and by COFEPRIS (Federal Commission for Protection against Sanitary Risks) on December 11, 2012. The study was conducted in accordance with the ethical principles of Helsinki and its amendments and the International Conference on Harmonisation for Good Clinical Practice Guideline.

The principal investigator informed the subjects of all processes, duration of the study, anticipated risks and discomfort it could entail, and an individual written informed consent was obtained prior to the initiation of the study procedures. The study was conducted from January to May of 2013.

\section{Inclusion/exclusion criteria}

Healthy Mexican adults aged 18 to 55 years and of either gender were eligible for inclusion in the study. Subjects were recruited from the volunteers' database at the Center of Pharmacological and Biotechnology Research (clinical unit) in Medica Sur Hospital, Mexico City, Mexico.

A clinical assessment was performed on each potential participant. Classification of the subjects as healthy was based on clinical health evaluation, which consisted of the following: a personal interview, a complete physical examination, vital signs (blood pressure (BP), heart rate, weight, height, temperature and respiratory rate); and diagnostic testing that included a 12-lead ECG, chest radiography, and laboratory hematology and chemistry panel, serological tests for hepatitis B, C and HIV antibodies, urinalysis, and a pregnancy test in women. Systolic and diastolic BP were measured with a qualified sphygmomanometer (Tycos; Welch Allyn, Skaneateles Falls, NY). The BP cuff was applied to the right arm and the reading was taken with the subject in a seated position. Candidates were excluded if laboratory values were significantly out of the reference range and/or if all tests had not been completed. Laboratory testing was performed at Medica Sur Laboratory, which has been certified by the Mexican government and the College of American Pathologists. The scope of the certifications included the tests relevant to this study. Before the enrollment of the participants, the laboratory data were reviewed by investigators. Selected candidates were compensated for their time dedicated to participation and transportation.

\section{Study design and drug administration}

A single-dose randomized-sequence, open-label, two-period, crossover design was used. A total of 26 subjects ( 21 men, 5 women) were admitted to the clinical site on the day before the study was begun and were randomly assigned by a pharmacist in the presence of quality assurance personnel in a 1:1 ratio, using a validated computergenerated table of random numbers, to one of the two sequences (test formulation containing moxifloxacin hydrochloride equivalent to $400 \mathrm{mg}$ of moxifloxacin [lot 234C0010; expiration date December 2014] followed by the reference formulation containing moxifloxacin hydrochloride equivalent to $400 \mathrm{mg}$ of moxifloxacin [lot XB104B4; expiration date May 2014], or vice versa). Randomization codes were concealed from all the investigators of the study.

To ensure reliable baseline plasma measurements, participants underwent a 10-hour overnight fast with an eight-day washout period, which exceeds the seven half-lives required by COFEPRIS.

Blood samples were drawn for baseline plasma determinations in the following way. A $18-\mathrm{G} \times 1.6$ in $(1.1 \times 30 \mathrm{~mm})$ peripheral intravenous catheter (BD-InSyte, Becton, Dickinson and Co., Sao Paulo, Brazil) was inserted in a suitable forearm vein and a $7.5 \mathrm{ml}$ blood sample was drawn into a heparin-treated vacuum tube (S-Monovette, Sarstedt AG \& Co., Nümbrecht, Germany).

Subjects were administered a single tablet of the test or the reference formulation with $250 \mathrm{ml}$ of water. Additional blood samples were drawn at $0.25,0.5,0.75,1,1.5,2,3,4,5,6,8,12,24,48$ and 72 hours after administration.

Plasma was obtained by centrifugation ( $1000 \mathrm{~g}$ for 15 minutes at $25^{\circ} \mathrm{C}$ ) and stored at $-75^{\circ} \mathrm{C} \pm 10^{\circ} \mathrm{C}$ until analyzed using HPLC. After an eight-day washout period, participants returned to the clinical unit, where the alternative formulation was administered keeping the same conditions as in the first treatment period.

Subjects were asked to refrain from water and food intake for three hours after study drug administration. Their diet, for each treatment period, consisted of three standardized meals $(2235.6 \mathrm{kcal} / \mathrm{d})$ at 3.25 , 6.5 and 12.75 hours after study drug administration.

During hospitalization, the subjects were under medical surveillance, and during the washout period participants maintained contact with the investigators to report any adverse events (AEs).

\section{Determination of moxifloxacin plasma concentrations}

Chemicals: Moxifloxacin hydrochloride (lot: F0H454, purity 95.9\%) reference standard was obtained from USP (Rockville, MD). All solvents were HPLC grade and all reagents were analytical grade.

Method and Sample Preparation: Moxifloxacin plasma levels were determined by using a HPLC method developed and validated by personnel of Biokinetics in Mexico City, Mexico. The method included the following: $250 \mu \mathrm{l}$ of plasma, $10 \mu \mathrm{l}$ of internal standard (telmisartan, $3000 \mu \mathrm{g} / \mathrm{ml}$ ) and $750 \mu \mathrm{l}$ of acetonitrile. These components were vortexed in a $2.0 \mathrm{ml}$ conical tube, (Sarstedt AG \& Co.) for one minute. The tube was centrifuged at $8000 \mathrm{rpm}$ for five minutes at room temperature $\left(25^{\circ} \mathrm{C}\right)$. The supernatant was separated and injected (volume of injection $=20 \mu \mathrm{l}$ ) into the chromatographic system (HPLC, Agilent Technologies, model 1100, Palo Alto, California).

\section{Chromatographic conditions}

Moxifloxacin concentrations were determined with a $150 \times 4.6$ mm internal-diameter column of $3.5 \mu \mathrm{m}$ particle size (Zorbax ${ }^{\circledR}$ Eclipse XDB-Phenyl, Agilent Technologies) and eluted with a mobile phase consisting of a mixture $(40: 60 \mathrm{v} / \mathrm{v})$ of an aqueous buffer solution (ammonium acetate, $10 \mathrm{mM} ; \mathrm{pH} 3.0 \pm 0.1$ ) and acetonitrile. The column temperature was $25^{\circ} \mathrm{C}$. Flow rate was maintained at $1 \mathrm{ml} /$ minute and the moxifloxacin was detected by a fluorescence detector (Agilent Technologies) set at an excitation wavelength of $296 \mathrm{~nm}$ and emission wavelength of $504 \mathrm{~nm}$. Typical retention times for moxifloxacin and the internal standard were two and four minutes, respectively. The peak area was measured for calculation of the peak area ratio of moxifloxacin with respect to the internal standard, and the concentration was calculated.

\section{Method validation}

The analytical method was validated according to Mexican [7] and international guidelines [8].

The selectivity of the method was tested by the analysis of blank human plasma samples from six different subjects, blank human (hemolyzed and lipemic) plasma samples, as well as anticoagulants (heparin), xanthines (caffeine and theobromine), and other drug 

Moxifloxacin 400 mg: An Open-Label, Randomized, Two-Period Crossover Comparison in Healthy Mexican Adult Volunteers. J Bioequiv Availab 6: 197-201. doi:10.4172/jbb.1000204

substances commonly used as analgesics (acetylsalicylic acid, ibuprofen, diclofenac, paracetamol and naproxen). No interferences were observed in the resulting chromatograms.

The range of the method was 0.07 to $2.0 \mu \mathrm{g} / \mathrm{ml}$, with lower limits of quantification (LLOQ) and of detection (LLOD) of 0.07 and $0.035 \mu \mathrm{g} /$ $\mathrm{ml}$, respectively. The method was found to be linear within this range of concentrations with a coefficient of determination of 0.99 . The intraassay $\% \mathrm{CV}$ and accuracy (relative error) for moxifloxacin were $1.22 \%$ to $3.23 \%$ and $-9.28 \%$ to $3.16 \%$, respectively, while the inter-assay $\% \mathrm{CV}$ and accuracy were $5.08 \%$ to $5.90 \%$ and $-2.25 \%$ to $-0.64 \%$. The absolute recovery was above $93.8 \%$.

Moxifloxacin in plasma was found to be stable after 24 hours at room temperature $\left(25^{\circ} \mathrm{C}\right)$, after three freeze-thaw cycles and after 16 weeks at $-75 \pm 5^{\circ} \mathrm{C}$.

Quality control (QC) samples were included in every analytical run to verify its performance. These QC samples were prepared at three different concentration levels (designated as low, $(0.15 \mu \mathrm{g} / \mathrm{ml})$, medium $(0.75 \mu \mathrm{g} / \mathrm{ml})$ and high $(1.5 \mu \mathrm{g} / \mathrm{ml}))$ of moxifloxacin independent of the calibration curve. This method was considered suitable by the investigators for the bioequivalence study of moxifloxacin.

\section{Tolerability}

Tolerability was determined using clinical assessment, monitoring of vital signs at baseline, after the drug administration during hospitalization, and at the end of the clinical stage of the study.

The subjects were interviewed (using open-ended questions) by the investigators during trial conduction and at the end of the clinical stage of the study, concerning the occurrence AEs. Subjects were asked to spontaneously report any $\mathrm{AE}$ to the investigators at any time during the study, including the washout period. Data for all AEs were recorded on a case-report form.

AEs that were life-threatening, led to death, hospitalization, disability, and/or medical intervention to prevent permanent impairment or damage, were considered serious.

\section{Pharmacokinetic and statistical analyses}

Sample size calculation [9] was based on the within-subject variability of moxifloxacin $\mathrm{C}_{\max }$ with a $\% \mathrm{CV}$ of $12.71 \%$ [10]. This calculation was performed considering the following values: $1-\beta=0.8$, $\alpha=0.05, \% \mathrm{CV}=12.71$, and an equivalence range of $80 \%$ to $125 \%$, yielded with a sample size of 10 subjects. In this study, a sample size of 26 subjects was used because, at the time the study was conducted, a minimum sample size of 24 subjects was required for bioequivalence studies by COFEPRIS.

Individual plasma concentration-time curves were constructed; $\mathrm{C}_{\max }$ and $\mathrm{T}_{\max }$ were directly obtained from these curves, the area under the plasma concentration-time curve from time baseline to the last measurable concentration $\left(\mathrm{AUC}_{0-t}\right)$ was calculated by a noncompartmental method using the trapezoidal rule. From the terminal log-decay phase, the elimination rate constant $\left(\mathrm{k}_{\mathrm{e}}\right)$ was estimated using linear regression, and the $t_{1 / 2}$ was estimated using the following equation [9]:

$\mathrm{t}_{1 / 2}=\ln 2 / \mathrm{k}$, where $\ln$ was defined as the natural logarithm. Extrapolation of AUC from baseline to infinity $\left(\mathrm{AUC}_{0-\infty}\right)$ was calculated as follows:

$\mathrm{AUC}_{0-\infty}=\mathrm{AUC}_{0-\mathrm{t}}+\left(\mathrm{C}_{\mathrm{t}} / \mathrm{k}_{\mathrm{e}}\right)$

Where, $C_{t}$ was the last measurable plasma concentration.
To assess the bioequivalence between the test and reference formulations, $\mathrm{C}_{\max }, \mathrm{AUC}_{0-\mathrm{t}}$ and $\mathrm{AUC}_{0-\infty}$ were considered as the primary variables. ANOVA for a $2 \times 2$ crossover design, using log-transformed data for these parameters, was carried out at the $5 \%$ significance level $(\alpha=0.05)$.

The $90 \%$ CIs of the geometric mean ratios (test/reference) of $\mathrm{C}_{\text {max }}$ $\mathrm{AUC}_{0-\mathrm{t}}$ and $\mathrm{AUC}_{0-\infty}$ were calculated using log-transformed data. The test and the reference formulations were considered bioequivalent if the $90 \%$ CIs of these parameters fell within a predetermined range of $80 \%$ to $125 \%$ and if the probability of exceeding these limits was $<0.05$. The probability of exceeding the $80 \%$ to $125 \%$ range was obtained using the two one-sided tests procedure described by Schuirmann [11]. All pharmacokinetic and parametrical-statistical analyses were performed using Win Nonlin Version 5 (Pharsight, Mountain View, California).

\section{Results}

A total of 26 subjects (21 men, 5 women; mean [SD] age, 34 [11] years [range, 19-51 years]; weight, 66.12 [6.11] kg [range, 53.10-77.70 $\mathrm{kg}$ ]; height, 1.67 [0.08] m [range, 1.47-1.81 m]; and body mass index [BMI], $23.74[1.55] \mathrm{kg} / \mathrm{m}^{2}$ [range, 20.04-25.96 kg/m²]) were enrolled and completed the clinical stage of the study.

\section{Pharmacokinetic parameters}

Mean plasma concentration-time curves of the two moxifloxacin formulations are shown in Figure 1. As can be seen in this figure, both curves look similar.

Table 1 shows the pharmacokinetic parameters $\left(C_{\max }, T_{\max }, t_{1 / 2}\right.$ $\mathrm{AUC}_{0-\mathrm{t}}$ and $\mathrm{AUC}_{0-\infty}$ ) for two data sets: the full data set and the reduced data set. The full data set consists of all of the subjects, including all of their moxifloxacin-concentration values. The reduced data set also consists of all of the subjects, with the single exception of an outlier concentration value $(1.74 \mu \mathrm{g} / \mathrm{mL}$ ) for one of the subjects (subject 12, when the reference formulation was administered).

This outlier was found at the last time point ( 72 hours), where concentration values below the LLOQwas expected. It is important to point out that an internal investigation was conducted and the results regarding the origin of the outlier were inconclusive. Therefore, it was decided to evaluate its potential impact on the estimation of the pharmacokinetic parameters, as well as on the results of the bioequivalence analysis.

In comparing both data sets, it can be seen that the effect of the outlier was to inflate the means and standard deviations of the $\mathrm{AUC}_{0-\infty}$ and $t_{1 / 2}$ values for the reference formulation. It can be noted that, when this outlier was excluded from the estimation (reduced data set), both means and standard deviations for these parameters were reduced to more comparable values between the two formulations.

No significant formulation or period-sequence effects (data not provided) were detected based on ANOVAof $\mathrm{C}_{\max }, \mathrm{AUC}_{0-\mathrm{t}}$ and $\mathrm{AUC}_{0-\infty}$ (for both data sets). The estimated intra-subject \%CVs for the full data set were $21.70,7.05$ and 25.99 , respectively; for the reduced data set they were $21.70,5.33$ and 7.42 .

Table 2 shows the bioequivalence statistics for both data sets (using the log-transformed data of $\mathrm{C}_{\text {max }}, \mathrm{AUC}_{0-\mathrm{t}}$ and $\mathrm{AUC}_{0-\infty}$ ): geometric mean ratios (test/reference) $(90 \% \mathrm{CI})$, the probabilities of exceeding the limits of acceptance for bioequivalence, and the power values of the test.

The 90\% CIs for moxifloxacin $\mathrm{C}_{m a x}, \mathrm{AUC}_{0-\mathrm{t}}$ and $\mathrm{AUC}_{0-\infty}$ for the full data set were $88.67 \%$ to $108.70 \%, 94.32 \%$ to $100.84 \%$, and $82.23 \%$ to 
Citation: Tamayo GM, Rosete A, Santillán RM, González J, Martínez CE, et al. (2014) Bioavailability of Two Tablet Formulations of a Single Dose of Moxifloxacin 400 mg: An Open-Label, Randomized, Two-Period Crossover Comparison in Healthy Mexican Adult Volunteers. J Bioequiv Availab 6: 197-201. doi:10.4172/jbb.1000204

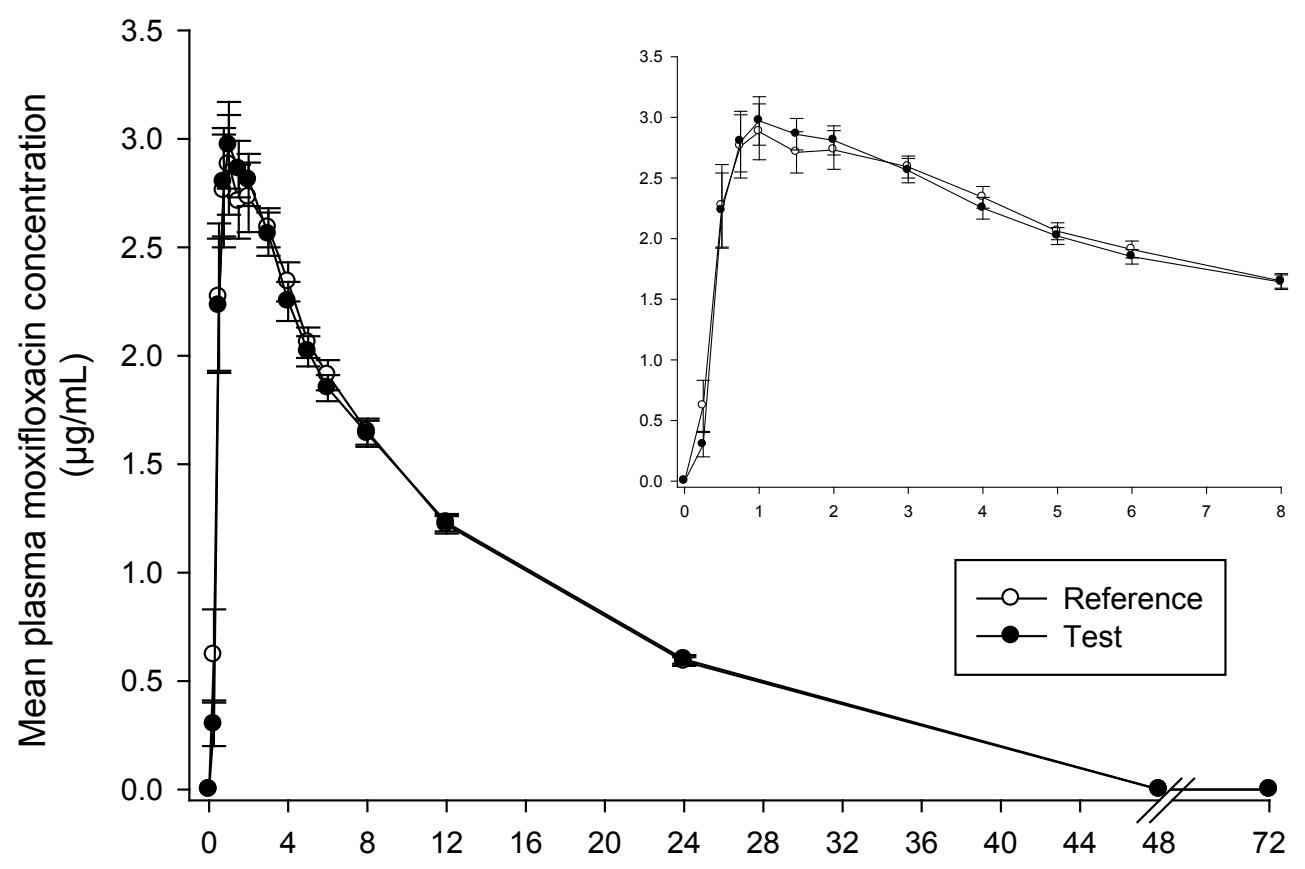

Time after study drug administration (h)

Figure 1: Mean plasma concentration-time curves after a single-dose administration of a test (trademark: Zinolox $4 \mathrm{G}^{\circledR}$, Laboratorios Liomont, S. A. de C. V., Mexico) and a reference (trademark: Avelox ${ }^{\circledR}$, Bayer de México, S. A. de C. V., Mexico City, Mexico) formulation of oral moxifloxacin 400 mg in healthy Mexican adult subjects $(n=26)$. Inset: mean (SE) over the first 8 hours after administation.

\begin{tabular}{|c|c|c|}
\hline Parameter & Test $^{*}$ & Reference $^{\dagger}$ \\
\hline \multicolumn{3}{|l|}{ Full data set } \\
\hline $\mathrm{C}_{\max }, \mu \mathrm{g} / \mathrm{ml}$ & $3.57(0.85)$ & $3.65(0.94)$ \\
\hline$A \cup C_{0-t}, \mu g \bullet h / m l$ & $33.91(5.40)$ & 34.87 (6.39) \\
\hline $\mathrm{AUC}_{0-\infty}, \mu \mathrm{g} \cdot \mathrm{h} / \mathrm{ml}$ & $43.73(6.67)$ & 52.96 (46.91) \\
\hline$T_{\max }, h$ & $1.15(0.75)$ & $1.51(1.19)$ \\
\hline$t_{1 / 2}, h$ & $11.25(1.58)$ & $14.12(15.79)$ \\
\hline \multicolumn{3}{|l|}{ Reduced data set } \\
\hline $\mathrm{C}_{\max }, \mu \mathrm{g} / \mathrm{ml}$ & $3.57(0.85)$ & $3.65(0.94)$ \\
\hline $\mathrm{AUC}_{0-\mathrm{t}}, \mu \mathrm{g} \cdot \mathrm{h} / \mathrm{ml}$ & $33.91(5.40)$ & $33.94(5.58)$ \\
\hline $\mathrm{AUC}_{0-\infty}, \mu \mathrm{g} \cdot \mathrm{h} / \mathrm{ml}$ & $43.73(6.67)$ & $43.31(7.35)$ \\
\hline$T_{\max }, h$ & $1.15(0.75)$ & $1.51(1.19)$ \\
\hline$t_{1 / 2}, h$ & $11.25(1.58)$ & $10.85(1.70)$ \\
\hline
\end{tabular}

The full data set includes all of the subjects, including all of their concentration values.

The reduced data set includes all of the subjects, with the single exception of the last concentration value (72-hours, reference formulation) for the subject identified as number 12.

$\mathrm{AUC}_{0-t}=\mathrm{AUC}$ from time 0 (baseline) to the last measurable concentration;

$\mathrm{AUC}_{0-\infty}=\mathrm{AUC}$ from baseline extrapolated to infinity.

'Trademark: Zinolox $4 \mathrm{G}^{\circledR}$ (Laboratorios Liomont, S. A. de C. V., Mexico City, Mexico)

†Trademark: Avelox ${ }^{\circledR}$ (Bayer de México, S. A. de C. V., Mexico City, Mexico).

Table 1: Pharmacokinetic parameters of a test and a reference formulation of oral moxifloxacin 400 mg after a single administration in healthy Mexican adult subjects (n $=26$ ). Values are mean (SD)

$104.81 \%$, respectively; and for the reduced data set they were $88.67 \%$ to $108.70 \%, 97.44 \%$ to $102.50 \%$ and $97.70 \% 104.82 \%$. For both data sets, all $90 \%$ CIs of the geometric mean ratios of the three parameters fell within the predetermined range of $80 \%$ to $125 \%$. All probability values were $<0.05$. All the power values were $>0.8$ (power $>80 \%$ ), suggesting that the sample size was sufficient for the purpose of the study.

These results indicated that the bioequivalence criteria were met, regardless of the exclusion of the outlier.

\section{Tolerability}

Seven of the 26 subjects reported a total of 12 AEs. The most commonly AE reported was dizziness ( $6 \mathrm{AEs}$ ), three after administration of the reference formulation and three after the administration of the test formulation.

Other AEs were headache (4 AEs), two after administration of the reference formulation, two after administration of the test formulation, and nausea ( 2 AEs), one after administration of the reference 
Citation: Tamayo GM, Rosete A, Santillán RM, González J, Martínez CE, et al. (2014) Bioavailability of Two Tablet Formulations of a Single Dose of Moxifloxacin 400 mg: An Open-Label, Randomized, Two-Period Crossover Comparison in Healthy Mexican Adult Volunteers. J Bioequiv Availab 6: 197-201. doi:10.4172/jbb.1000204

\begin{tabular}{|c|c|c|c|c|}
\hline \multirow[t]{2}{*}{ Parameter } & \multirow{2}{*}{$\begin{array}{c}\text { Geometric Mean Ratio, \% } \\
(90 \% \mathrm{Cl})\end{array}$} & \multicolumn{2}{|c|}{$\begin{array}{l}\text { Probability of } \\
\text { Exceeding Limits of } \\
\text { Acceptance }\end{array}$} & \multirow[t]{2}{*}{ Power } \\
\hline & & $P<80 \%$ & $P>125 \%$ & \\
\hline \multicolumn{5}{|l|}{ Full data set } \\
\hline $\mathrm{C}_{\max }$ & $98.17(88.67,108.70)$ & $<0.001$ & $<0.001$ & 0.97 \\
\hline $\mathrm{AUC}_{0-\mathrm{t}}$ & $97.53(94.32,100.84)$ & $<0.001$ & $<0.001$ & 1.00 \\
\hline$A \cup C_{0-\infty}$ & $92.84(82.23,104.81)$ & 0.023 & $<0.001$ & 0.92 \\
\hline \multicolumn{5}{|c|}{ Reduced data set } \\
\hline $\mathrm{C}_{\max }$ & $98.17(88.67,108.70)$ & $<0.001$ & $<0.001$ & 0.97 \\
\hline $\mathrm{AUC}_{0-\mathrm{t}}$ & $99.94(97.44,102.50)$ & $<0.001$ & $<0.001$ & 1.00 \\
\hline$A \cup C_{0-\infty}$ & $101.19(97.70,104.82)$ & $<0.001$ & $<0.001$ & 1.00 \\
\hline
\end{tabular}

The full data set includes all of the subjects, including all of their concentration values.

The reduced data set includes all of the subjects, with the single exception of the last concentration value (72-hours, reference formulation) for the subject identified as number 12.

$\mathrm{AUC}_{0-\mathrm{t}}=\mathrm{AUC}$ from time 0 (baseline) to the last measurable concentration

$A \cup C_{0-\infty}^{0-t}=A \cup C$ from baseline extrapolated to infinity.

"Values were calculated from the natural logarithm-transformed data.

†Trademark: Zlinolox $4 \mathrm{G}^{\circledR}$ (Laboratorios Liomont, S. A. de C. V., Mexico City, Mexico)

抽ademark: Avelox ${ }^{\circledR}$ (Bayer de México, S. A. de C. V., Mexico City, Mexico).

Table 2: Geometric mean ratios $(90 \% \mathrm{Cl})$ of pharmacokinetic parameters the probabilities of exceeding the predetermined limits of bioequivalence $(80 \%$ $125 \%$ ), and the power test results from a single-dose administration of a test ${ }^{\dagger}$ and a reference ${ }^{\ddagger}$ formulation of oral moxifloxacin $400 \mathrm{mg}$ in healthy Mexican adult subjects.

formulation, one after administration of the test formulation. Most were classified as mild and two as moderate (dizziness). All of the AEs were spontaneously resolved under medical surveillance.

None of the AEs was considered serious. In addition, all of these types of AEs have been previously reported with moxifloxacin treatment [12].

\section{Discussion}

For both data sets, all of the $90 \%$ CIs of the geometric mean ratios of the pharmacokinetic parameters $\left(\mathrm{C}_{\max }, \mathrm{AUC}_{0-\mathrm{t}}\right.$ and $\left.\mathrm{AUC}_{0-\infty}\right)$ were found to be within the predetermined range of bioequivalence $(80 \%$ $125 \%$ ) and the Schuirmann tests (i.e., probability of exceeding limits of acceptance) found all of the probability values to be $<0.05$; these results satisfy the accepted regulatory requirements to assume bioequivalence.

Although the presence of the outlier did not affect the conclusion of bioequivalence, it did inflate the estimates of $\mathrm{AUC}_{0-\infty}$ and $\mathrm{t}_{1 / 2}$ for the reference formulation, as well as for both the intra-subject $\% \mathrm{CV}$ values and the width of the $90 \% \mathrm{CI}$ of $\mathrm{AUC}_{0-\mathrm{t}}$ and $\mathrm{AUC}_{0-\infty}$. Hence, it is import to consider the impact of outliers on the data analysis.

Both formulations were well tolerated, in view of that fact that none of the reported AEs by seven subjects was considered serious and all been previously reported.

\section{Limitations}

As with any clinical trial, and in particular with most bioavailability studies, the current study had some limitations that should be considered. First, this was an open-label study, so it might not objectively address the safety profiles of the formulations tested, but distribution of AEs was the same for both formulations.

The data were obtained from healthy adult subjects, in accordance with regulatory requirements (COFEPRIS), within a specific age range, who were administered a single dose; the $\mathrm{PK}$ parameters of moxifloxacin might differ in target populations. For example, differences in absorption, metabolism and excretion of moxifloxacin might exist in patients, with respect to healthy subjects. Thus, the results of this study might not be generalizable to a target ill population.

In addition, this study was conducted under fasting conditions because the bioavailability of moxifloxacin has been reported not to be affected by the concomitant intake of food [6].

Because of the limited data in the present study (small sample size, single dose, healthy subjects, age range, and fasting conditions), we are unable to predict the response of the drug at any time following alternative doses and/or administration intervals with respect to the present data set. Further studies are needed to compare the test formulation with the reference formulation in Mexican patient groups. The results of this study might serve as a reference for future controlled studies of moxifloxacin in a Hispanic population.

\section{Conclusions}

In this small study of healthy, fasting, Mexican adult subjects, single doses of oral moxifloxacin $400 \mathrm{mg}$ met the Mexican (COFEPRIS) regulatory requirements to assume bioequivalence based on the rate and extent of absorption. Both formulations were well tolerated.

\section{Acknowledgements}

This research and its publication were supported by Laboratorios Liomont S.A. de C.V., Mexico City, Mexico. The authors have indicated that they have no other conflicts of interest regarding the content of the article.

\section{References}

1. Barman-Balfour JA, Wiseman L (1999) Moxifloxacin. Drugs 57: 363-373

2. Caeiro JP, lannini PB (2003) Moxifloxacin (Avelox): a novel fluoroquinolone with a broad spectrum of activity. Expert Rev Anti Infect Ther 1: 363-370.

3. Ballow C, Lettieri J, Agarwal V, Liu P, Stass H, et al. (1999) Absolute bioavailability of moxifloxacin. ClinTher 21: 513-522.

4. Sullivan JT, Woodruff M, Lettieri J, Agarwal V, Krol GJ, et al. (1999) Pharmacokinetics of once-daily oral dose of moxifloxacin (Bay 12-8039), a new enantiomerically pure 8-methoxy quinolone. Antimicrob Agents Chemother 43 : 2793-2797.

5. Stass H, Dalhoff A, Kubitza D, Schühly U (1998) Pharmacokinetics, safety and tolerability of ascending single doses of moxifloxacin, a new 8-methoxy quinolone, administered to healthy subjects. Antimicrob Agents Chemother 42 : 2060-2065.

6. Lettieri J, Vargas R, Agarwal V, Liu P (2001) Effect of food on the pharmacokinetics of a single oral dose of moxifloxacin $400 \mathrm{mg}$ in healthy male volunteers. Clin Pharmacokinet 40: 19-25.

7. COFEPRIS, Federal Commission for the Protection against Sanitary Risks (1999) Tests and procedures to prove that a medication is interchangeable [in Spanish]. Official Mexican Standard NOM 177-SSA1-1998.General Directorate of Standards, Mexico City, Mexico.

8. CDER, Center for Drug Evaluation and Research (2001). Guidance for Industry Bioanalytical method validation.

9. Chow SC, Liu JP (2000) Design and Analysis of Bioavailability and Bioequivalence Studies. (2ndedn), Marcel Dekker, New York.

10. Heads of Medicines Agencies (2009) Public Assessment Report of Infekt 400 mg film-coated tablets, moxifloxacin hydrochloride. DK/H/1742/001/MR.

11. Schuirmann DJ (1987) A comparison of the two one-sided tests procedure and the power approach for assessing the equivalence of average bioavailability. $J$ Pharmacokinet Biopharm15:657-680

12. National Institutes of Health (2013) Moxifloxacin 